\title{
Compressed Sensing Channel Estimation with FBMC-Based Large Scale MIMO using Gaussian Mixture Learning
}

\author{
T. Ravi Babu ${ }^{1}$, C. Dharma Raj ${ }^{2}$, CH. Swapna priya ${ }^{3}$, V. Adinarayana ${ }^{4}$ \\ ${ }^{1,2}$ Department of ECE, GITAM Institute of Technology, (GIT) GITAM, Visakhapatnam, India. \\ ${ }^{1}$ razh1976@gmail.com.2 dharmaraj.cheruku@gitam.edu. \\ ${ }^{3}$ Department of CSE, Vignan's Institute of information Technology, \\ Visakhapatnam, India. swapnachsp@gmail.com. \\ ${ }^{4}$ Department of ECE, AVANTHI Institute of Technology, \\ Visakhapatnam, India. joy.adi2011@gmail.com
}

\begin{abstract}
Filter Bank based Multi-Carrier technique is a viable solution to obtain higher spectral efficiency, out-of-band emissions, higher energy and extended data rates over Orthogonal Frequency Division Multiplexing (OFDM) of future $5 \mathrm{G}$ applications. To achieve the significant gains at the massive MIMO-FBMC system, low complexity compressed sensing method serves along with the generalized approximate message passing (GAMP) algorithm is proposed into time-domain scenarios. However, the sparsity estimation between the communicating channels, non-zero vector distribution can be taking into consideration the Gaussian mixture accordingly, learn their characteristics towards the expectation-maximization procedure. The results of the simulation have proved the performance of the proposed estimation approach of channel keeping with minimum pilot overhead and developed exceptional Bit Error Rate (BER) performance of the system.
\end{abstract}

Key words: Approximate message passing, Compressed sensing, Channel estimation, Gaussian Mixture, Massive MIMO-OFDM.

\section{INTRODUCTION}

The huge demand of high data rates in 5G technology, the conventional MIMO antenna system needs to extend massive MIMO to increase the potential support of spectral efficiency, reliability \& overall system capacity $[1,5]$. The subsequent work analyzed on massive MIMO systems [2], to provide higher spectral efficiency using simple techniques of transmission and reception. Because of more number of transmitters and receivers are using at massive MIMO systems, the CSIT acquisition resembles as a most challenging problem [15].

In FDD systems no channel reciprocity attained due to usage different frequencies in uplink and downlink transmission. Usually, estimating pictures of the channel requires a specific sequence to train provided that information of the previous outputs received in course of training on standard procedure is available. Additional feedback is required for getting the statistics of nonstationary MIMO. To circumvent this situation, a novel technique of CSI determination along with feedback strategy giving accurate reliable CSIT having reduced complexity as well as overhead is desirable. Compressed sensing is offers suitable method for estimating short sequence type of sparse with unknown statistics $[11,12]$. In this paper, combined of LS and CS techniques are used to obtain estimation in FDD $[15,16]$ having various of sparse and dense vectors. Due to improved recovery performance methods in [3], Bayesian estimation scores over other equivalent methods; it can increase monitoring of fractional space of the channel by reducing pilot overhead.

The Massive MIMO-FBMC is preeminent and supportive technology to $5 \mathrm{G}$ wireless applications that has to maintain excellent data rate and accuracy. To achieve these eminent properties, knowledge of channel information is a most challenging issue in massive MIMO- FBMC systems, therefore, it is necessary to apply relevant estimation techniques to channels between all transmitting and receiving antennas accordingly. In general, communicating channels are inherently sparse; however, the majority of channels viewed as zero coefficients at channel impulse response (CIR). With a focus on channel sparsity, we implemented the compressed sensing method to characterize the channel properties of the proposed model [1].

The sparsity adaptive matching pursuit (SAMP) provides high performance at a wide range of practical applications without channel sparsity. However, there is inconsistency between convergence speed and recovery accuracy because SAMP has maintained a constant step size [2, 3]. OFDM is one of the modulating systems that provide to mitigate interference and cross talk resulting from the conversion of the serial data stream into parallel data stream at different frequencies. OFDM massive MIMO compressed sensing based channel estimation is set as sparse and dense vectors. 
These vectors are a combination of zero and nonzero vectors respectively. On the perfect sparse recovery, the sparse signal is reconstructed through the support of the LS technique [12].

The expectation-maximization (EM) steps are established to accomplish quantities of estimation following a Gaussian mixture model. Furthermore, the generalized approximate message passing (GAMP) which is an active algorithm in i.i.d distributed random signal [14] is exploiting to develop the expectation step and also mitigate computational complexity. The fast iterative truncation algorithm (FITRA) which is for sparse representation that was In current work, we established and compared with a renewed compressed sensing aided over Gaussian mixture algorithms for down link massive MIMO-FBMC system with a reference of ZF pre-coding technique respectively. To estimate the error performance, the truncated and Bernoulli Gaussian mixture procedures are considered and entrusted to the unknown signal.

Simulated results found that the suggested algorithms provide a substantial improvement in terms of computational difficulties. The remainder part of this work is partitioned as per the following. Second Section discusses down link system model. The third and forth section covers training sequence design and the principle of estimation of the proposed technique, the fifth and sixth section addresses the random noise with GAMP and covers GAMP based FBMC channel estimation for comparing various OMP approaches, seventh section includes discussions of simulation results, and finally seventh section concludes.

\section{SYSTEM MODEL}

Consider the transmitter, receiver antennas $N_{T}$ and $N_{R}$ respectively, and $M$ subcarriers through time-domain MIMO-FBMC system. In addition to a set of parallel data symbols are transmitted through a bank of synthesis and analysis filters. The prototype filter decides the localization in time-domain and frequency-domain of the generated waveform. The frequency-domain data symbols to be transmitted on the $m^{\text {th }}$ subcarrier of the $k^{\text {th }}$ time constant in $i^{\text {th }}$ transmit antenna is $x_{m}^{i}(k)$.

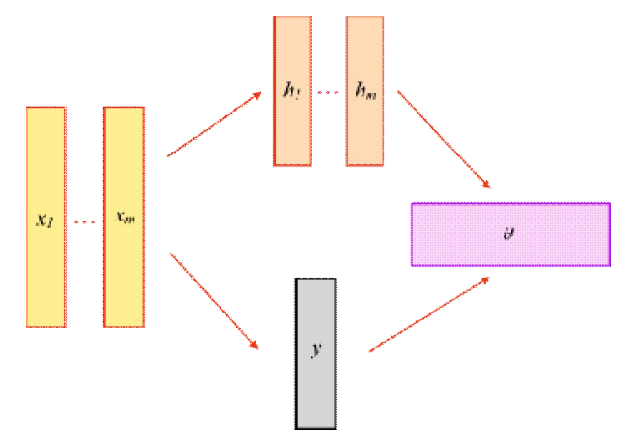

Figure 1: Outline of compressive learning.
The $x_{m}^{i}(k)$ is defined as $d_{m}^{i}(k) \theta_{m}^{i}(k)$, where $d_{m}^{i}(k)$ and $\theta_{m}^{i}(k)$ are real data and real orthogonality between adjacent grids, respectively. The transmitted signal represented in time-domain as follows:

$$
s^{i}(n)=\sum_{k=-\infty}^{\infty} \sum_{m=0}^{M-1} x_{m}^{i}(k) p(n) e^{j \frac{2 \pi}{M} m(n-D)}
$$

Here $p(n)$ represents the proto type filter.

The received signal can be described with variance $\sigma^{2}$ behalf of $j^{\text {th }}$ antenna as given by

$$
y^{j}(n)=\sum_{i=1}^{N_{T}} s^{i}(n) \times h_{i j}(n)+w^{j}(n)
$$

The desired time-frequency decoded signal from filter bank output at $q^{\text {th }}$ subcarrier and $t^{\text {th }}$ time constant in the $j^{\text {th }}$ antenna is described by

$$
y_{q}^{j}(t)=\sum_{n=-\infty}^{\infty} r^{j}(n) \times p\left[n-t \frac{M}{2}\right] e^{-j \frac{2 \pi}{M} q\left(n-t \frac{M}{2}-D\right)}
$$

Substitute the (2) into (3), we can get

$$
\begin{aligned}
y_{q}^{j}(t)= & \sum_{l=0}^{L_{h}-1} A_{q, l}^{(i)} h(l)+ \\
& \sum_{n=-\infty}^{\infty} w^{j}(n) \times p\left[n-t \frac{M}{2}\right] e^{-j \frac{2 \pi}{M} q\left(n-t \frac{M}{2}-D\right)}
\end{aligned}
$$

Here

$$
\begin{aligned}
A_{q, l}^{(i)}=\sum_{n=-\infty}^{\infty} & \sum_{l=0}^{L_{h}-1} \sum_{i=1}^{N_{T}} \sum_{k=-\infty}^{\infty} \sum_{m=0}^{M-1} d_{m}^{i}(k) \theta_{m}^{i}(k) F_{n, l, k, t, m, q} \\
F_{n, l, k, t, m, q}= & p\left[n-l-k \frac{M}{2}\right] \\
p & {\left[n-t \frac{M}{2}\right] e^{-j \frac{2 \pi}{M} q(n-D)(m-q)} e^{-j \pi(m k-q t)} }
\end{aligned}
$$

\section{GAUSSIAN MIXTURE GENERALIZED AMP}

To address the random Gaussian noise, generalized AMP (GAMP) algorithm, proposal was made by Rangan [7]. This proposed approach need not require knowledge about $p_{X}($.$) and the postulated noise variance, nevertheless it$ presents great recovery performance, that need to know about these postulated information. In this Gaussian-mixture GAMP algorithm, we consider the coefficient in $\boldsymbol{x}=$ $\left[x_{1}, x_{2}, \ldots, x_{N}\right]^{T}$ is approaching to the i.i.d distribution with marginal probability density function can be expressed as

$$
\begin{aligned}
p_{X}(x ; \lambda, \boldsymbol{\omega}, \boldsymbol{\theta}, \phi) & =(1-\lambda) \delta(x) \\
& +\lambda \sum_{l=1}^{L} \omega_{l} \mathcal{N}\left(x ; \theta_{i}, \phi_{i}\right)
\end{aligned}
$$


Where $\delta(\cdot)$ function denotes Dirac delta identity and $\lambda$ is the percentage of sparsity rate. And $(\omega, \theta, \phi)_{k}$ coefficients are expressed as the weight, mean, and variance of $k^{\text {th }}$ Gaussian Mixture components respectively. In the remainder part $\sum_{i=1}^{L} \omega_{i}=1$ and zero mean and variance $\psi$ is used as noise. Here, the postulated parameters of GM-GAMP $\boldsymbol{q} \triangleq$ $(\lambda, \boldsymbol{\omega}, \boldsymbol{\theta}, \phi, \psi)$ are taken into consideration as known and fixed variables. Firstly, carry out the conditional distribution $p_{Y \mid Z}\left(y_{n} \mid z_{n} ; \boldsymbol{q}\right)$; the expanded postulated approximation can be expressed as

$$
p_{Z \mid \boldsymbol{Y}}\left(z_{n} \mid \boldsymbol{y} ; \hat{p}_{n}, \mu_{n}^{p}, \boldsymbol{q}\right) \triangleq \frac{p_{Y \mid Z}\left(y_{n} \mid z_{n} ; \boldsymbol{q}\right) \mathcal{N}\left(z_{n} ; \hat{p}_{n}, \mu_{n}^{p}\right)}{\int_{z} p_{Y \mid Z}\left(y_{n} \mid z ; \boldsymbol{q}\right) \mathcal{N}\left(z ; \hat{p}_{n}, \mu_{n}^{p}\right)}
$$

The moments of above density function under AWGN assumption is as

$$
\begin{aligned}
& E_{Z \mid Y}\left\{z_{n} \mid \boldsymbol{y} ; \hat{p}_{n}, \mu_{n}^{p}, \boldsymbol{q}\right\}=\hat{p}_{n}+\frac{\mu_{n}^{p}}{\mu_{n}^{p}+\psi}\left(y_{n}-\hat{p}_{n}\right) \\
& \left.\operatorname{var}_{Z \mid \boldsymbol{Y}}\left\{z_{n} \mid \boldsymbol{y} ; \hat{p}_{n}, \mu_{n}^{p}, \boldsymbol{q}\right\}=\frac{\mu_{n}^{p} \psi}{\mu_{n}^{p}+\psi}\right)
\end{aligned}
$$

Secondly for computing the conditional distribution $p_{Y \mid Z}\left(x_{i} \mid \boldsymbol{y} ; \boldsymbol{q}\right) ;$ the expanded postulated approximation can be expressed as

$p_{X \mid \boldsymbol{Y}}\left(x_{i} \mid \boldsymbol{y} ; \hat{r}_{i}, \mu_{i}^{r}, \boldsymbol{q}\right) \triangleq \frac{p_{X}\left(x_{i} ; \boldsymbol{q}\right) \mathcal{N}\left(x_{i}, \hat{r}_{i}, \mu_{i}^{r}\right)}{\int_{X} p_{X}(x ; \boldsymbol{q}) \mathcal{N}\left(x, \hat{r}_{i}, \mu_{i}^{r}\right)}$

To achieve subsequent approximation of GM-GAMP substitute the postulated parameters (3) into (8) and simplified expression can be given as

$$
\begin{aligned}
& p_{X \mid \boldsymbol{Y}}\left(x_{i} \mid \boldsymbol{y} ; \hat{r}_{i}, \mu_{i}^{r}, \boldsymbol{q}\right)= \\
& {\left[(1-\lambda) \delta\left(x_{i}\right)+\lambda \sum_{l=1}^{L} \omega_{l} \mathcal{N}\left(x_{i} ; \hat{r}_{i}, \mu_{i}^{r}\right)\right] \frac{\mathcal{N}\left(x_{i} ; \hat{r}_{i}, \mu_{i}^{r}\right)}{\varsigma_{i}}} \\
& =\left[\left(1-\pi_{i}\right) \delta\left(x_{i}\right)+\pi_{i} \sum_{l=1}^{L} \bar{\beta}_{i, l} \mathcal{N}\left(x_{i} ; \gamma_{i, l}, v_{i, l}\right)\right]
\end{aligned}
$$

In (11) $\pi_{i}$ indicates posterior support probability values, $\operatorname{Pr}\left\{x_{i} \neq 0 \mid \boldsymbol{y} ; \boldsymbol{q}\right\}$ of GM-GAMP approximation. The normalized factor in (11) can be written as

$$
\begin{aligned}
\varsigma_{i} \triangleq \int_{X} p_{X}(x ; \boldsymbol{q}) \mathcal{N}\left(x, \hat{r}_{i}, \mu_{i}^{r}\right)=(1-\lambda) \mathcal{N}\left(0 ; \hat{r}_{i}, \mu_{i}^{r}\right) \\
+\lambda \sum_{l=1}^{L} \omega_{l} \mathcal{N}\left(0 ; \hat{r}_{i}-\theta_{l}, \mu_{i}^{r}+\phi_{l}\right)
\end{aligned}
$$

Both (13) and (14) can be derived from (13a) through Gaussian probability density function multiplication rule. In (14) the following dependent variables can be given by

$$
\begin{aligned}
& \pi_{i} \triangleq \frac{1}{1+\left(\frac{\sum_{l=1}^{L} \beta_{i, l}}{(1-\lambda) \mathcal{N}\left(0 ; \hat{r}_{i}, \mu_{i}^{r}\right)}\right)^{-1}} \\
& \gamma_{i, l} \triangleq \frac{\frac{\hat{r}_{i}}{\mu_{i}^{r}}+\theta_{l} / \phi_{l}}{\frac{1}{\mu_{i}^{r}}+1 / \phi_{l}} \text { and } v_{i, l} \triangleq \frac{1}{\frac{1}{\mu_{i}^{r}}+1 / \phi_{l}} \\
& \beta_{i, l} \triangleq \lambda \omega_{l} \mathcal{N}\left(0 ; \hat{r}_{i} ; \theta_{l}, \mu_{i}^{r}+\phi_{l}\right) \text { and } \bar{\beta}_{i, l} \triangleq \frac{\beta_{i, l}}{\sum_{l=1}^{L} \beta_{i, k}}
\end{aligned}
$$

To achieve effectiveness of GAMP approximation along with L-term Gaussian Mixture is used to overcome difficulties on realistic implementations. Using Bayesian parameter estimate method through AMP algorithm [4] provides accurate approximation that involved with centrallimit-theorem, together with independent identically distributed zero-mean Gaussian $\boldsymbol{A}$.

\section{GAMP-BASED FBMC CHANNEL ESTIMATION}

Let combine all receiving antennas to justify in matrix form as

$$
\mathbf{y}=\mathbf{A h}+\mathbf{z}
$$

Where $\mathbf{A}$ and $\mathbf{h}$ is the vector matrix of the channel, having a dimension of $N_{R} \times M$, with $\mathbf{A}=\mathbf{I}_{\mathrm{N}_{\mathrm{R}}} \times\left[\mathbf{A}^{(1)}, \mathbf{A}^{(2)}, \ldots \mathbf{A}^{\left(N_{T}\right)}\right]$ and $\mathbf{h}=\left[\mathbf{h}_{11}^{T}, \mathbf{h}_{12}^{T}, \ldots \mathbf{h}_{N_{T} N_{R}}^{T}\right]^{T}$ respectively. Consider the estimation of $P(P \leq M)$ pilot subcarriers from compressed sensing theory, rewrite the (14) yields,

$\mathbf{Y}_{\mathrm{P}}=\mathbf{A}_{\mathrm{P}} \mathbf{h}+\mathbf{Z}_{\mathrm{P}}$

Here $\mathbf{y}_{\mathrm{P}}$ denotes respected frequency domain channel vector and $\mathbf{A}_{\mathrm{P}}$ is the $P$ rows of $\mathbf{A}$ of channel vector.

The PDF of Gaussian prior distribution for modeling of the sparsity of the channel, the CIR taps, $h_{n}$ are identified as follows:

$$
p_{h_{n}}(h)=\frac{K}{L_{h}} C N\left(h ; \mu_{l} ; \sigma_{l}\right)+\left(1-\frac{K}{L_{h}}\right) \delta(h)
$$

Here $\frac{K}{L_{h}}$ denotes sparsity rate that are non-zero CIR coefficients and $\quad C N\left(h ; \mu_{l} ; \sigma_{l}\right)=\left(\pi \sigma_{n}\right)^{-1} e^{\left(-\sigma_{n}^{-1}\left|h-\mu_{n}\right|^{2}\right)}$ expressed as complex Gaussian probability density function. The CIR coefficients can be found from pilot observations of $P N_{R}$, the estimated values are expressed by

$\hat{h}_{n}=\sum_{h_{n}} h_{n} p\left(h_{n} \mid Y_{p}\right)$ 


\section{CS-BASED FBMC CHANNEL ESTIMATION}

In this section describes the CS based massive MIMO channel estimation model of $n^{\text {th }}$ antenna that is denoted as

$\boldsymbol{y}_{P}=\sum_{i=1}^{L} \operatorname{diag}\left(\mathbf{X}_{P}\right) \mathbf{F}_{P} \mathbf{h}_{n}+\boldsymbol{z}_{P}$

To estimate unknown vector $\mathbf{h}_{n}$ of massive MIMO system, the proposed CS recovery algorithm is employed. In many conventional greedy algorithms requires priori information at receiver side. Moreover, the greedy iteration schemes suffer with inaccuracy and more computational complexity $[12,15]$. Either of these two schemes has been successfully detected the fading channel information [14].

Algorithm 1:

CS-Based Channel Estimation of Massive MIMO-FBMC

Input: The first formulations $h_{n}, \tau_{n}^{h}$ and $\mathrm{I}_{n}$ are selected.

1. Initialize $\hat{h}_{n}(0)=\tau_{n}^{h}(0)=t=\hat{s}(-1)=0$

2. $\tau_{m}^{p}(t)=\sum_{m}\left|a_{m n}\right|^{2} \tau_{n}^{h}(t) \quad$ and $\quad \hat{p}_{m}(t)=$ $\sum_{n} a_{m n} \hat{h}_{n}(t)-\tau_{m}^{p}(t) \hat{s}_{m}(t-1)$; compute $m$ value for each step of linear output;

3. $t=t+1$

4. $\tau_{m}^{s}(t)=1 / \tau_{m}^{s}(t)+\sigma^{2} \quad$ and $\quad \hat{s}_{m}(t)=\left(y_{m}-\right.$ $\left.\hat{p}_{m}(t)\right) / \tau_{m}^{p}(t)+\sigma^{2}$; compute $m$ value for each step of nonlinear output;

5. $\tau_{n}^{r}(t)=1 / \sum_{m}\left|a_{m n}\right|^{2} \tau_{m}^{s}(t)$ and $\hat{r}_{n}(t)=\hat{h}_{n}(t)+$ $\tau_{n}^{r}(t) 1 / \sum_{m} a_{m n} \hat{s}_{m}(t)$; compute $n$ value for each step of linear input;

6. $\hat{h}_{n}(t+1)=\sum_{l \in[K]} b_{l} p_{l} \quad$ and $\quad \tau_{n}^{h}(t+1)=$ $\sum_{l \in[K]}\left|b_{l}\right|^{2} p_{l}-\left|\hat{h}_{n}(t+1)\right|^{2}$; compute $n$ value for each step nonlinear input;

7. $p_{l}=\phi\left(b_{l} ; \hat{r}_{n}(t), \tau_{n}^{r}(t)\right) K P_{b}$

8. $P_{b}=$

$\left(1-\frac{K}{L_{h}}\right) \phi\left(0 ; \hat{r}_{n}(t), \tau_{n}^{r}(t)\right)+$

$\frac{1}{K} \sum_{l \in[K]} \phi\left(b_{l} ; \hat{r}_{n}(t), \tau_{n}^{r}(t)\right)$

9. $\phi\left(u ; \hat{r}_{n}(t), \tau_{n}^{r}(t)\right)=\exp \left(-\left|u-\hat{r}_{n}(t)\right|^{2} / \tau_{n}^{r}(t)\right)$

End while

Output: estimate the CIR $\hat{h}_{n}(t+1)$

However, the proposed CS recovery algorithm need not consider the channel level as a prior information. This iteration is in accordance with the Partial common support information (PCSI) and depending on an iteration threshold. The PCSI of the $n^{\text {th }}$ receive antenna is expressed by

$\mathbf{I}_{n}=\sum_{n=1}^{L} \mathbf{I}_{n}+(n-1) L$

In the CS based channel estimation algorithm the following notations are involved.
Updated measurement matrix, Observation vector, column index, iteration threshold, updated index, estimated channel vector, and residual are expressed by $\mathrm{A}_{t}, Y_{P}, \lambda_{t}, \varepsilon, \Lambda_{t}, \mathrm{r}_{t}, \hat{\mathbf{h}}_{n, t}$ respectively [5]. There are two major challenges with applying OMP to massive data [13]. Firstly, the computation complexity and an iteration storage cost are relatively large and secondly, the single coefficient selection simultaneously requires the corresponding $k$ iterations to estimate with $k$ coefficient of $\boldsymbol{q}$. whenever, the $k$ iterations are increases that leads impracticably slow down its performance.

CS based schemes (Static or Dynamic LS, OMP, CSAided and BCS), the number of iterations are based on sparsity level of the channel $[3,6,11]$ whereas the proposed method closes iteration only when the residual is on the threshold of 0 . Consequently, the recovery accuracy can be assured. Moreover, when acquired accurate partial common support information, the number of iterations are limited; leads decrease in computational complexity of the proposed method.

To achieve this objective, we propose a Gaussian mixture (GM) methodical approach and afterwards by utilizing the expectation-maximization (EM) method $[8,14]$ to determine the noise variance and GM parameters. However, no need for concern quantities for the EM updates because these computations made by suggested GAMP algorithm to decrease computational complexity [9]. The proposed generalized EM-TGM-AMP and EM-BGM-AMP can be effectively addressing at parametric estimation with i.i.d zero-mean Gaussian.

\section{DISCUSSIONS AND SIMULATION RESULTS}

The efficiency of proposed truncated Gaussian mixture EM-GAMP and Gaussian Bernoulli EM-GAMP algorithm can be compared in accordance with obtained results of zero-forcing (ZF) pre-coding OMP and CS-Aided approach.

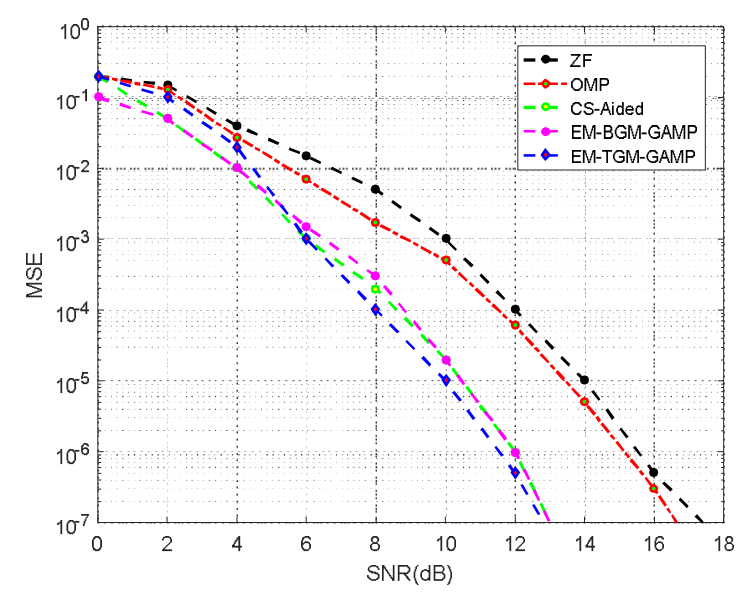

Figure 2: MSE versus SNR recovery performance to compressed sensing based OFDM with various schemes. 
Figure 2 has presented the OFDM comparison of various schemes of NMSE performance with known error value of $k_{s}^{e}$ (assume estimate channel coefficient $k_{s}=2$ ). The estimation methods are employing with the previous support information shows the poor performance upon the unbalanced parameter arises, whereas the quality of support information is trivial to the proposed approaches. Because the imperfect channel coefficients are evaluated by a compressed sensing (CS) algorithm and has low mismatched influence. In addition, if erroneous coefficients are selected by this method that clipping step can terminate the impact of erroneous coefficients. In addition, proposed and CS-Aided methods can be maintained the constant support information.

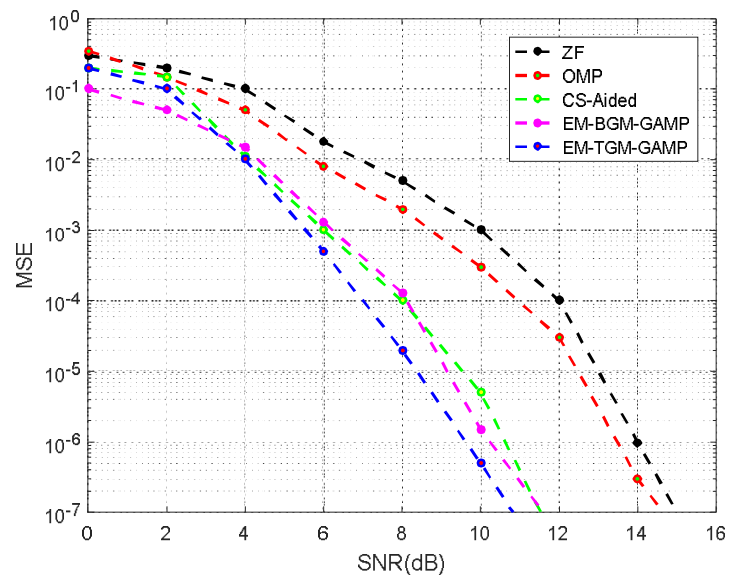

Figure 3: MSE versus SNR recovery performance to compressed sensing based FBMC with various schemes.

Figure 3 indicates the FBMC performance of MSE versus SNR with various schemes.. Since, the noise level of CS algorithms are fundamentally sensitive, the estimation methods are employing with CS, including the considered approaches, yields great outcome in range of SNR.

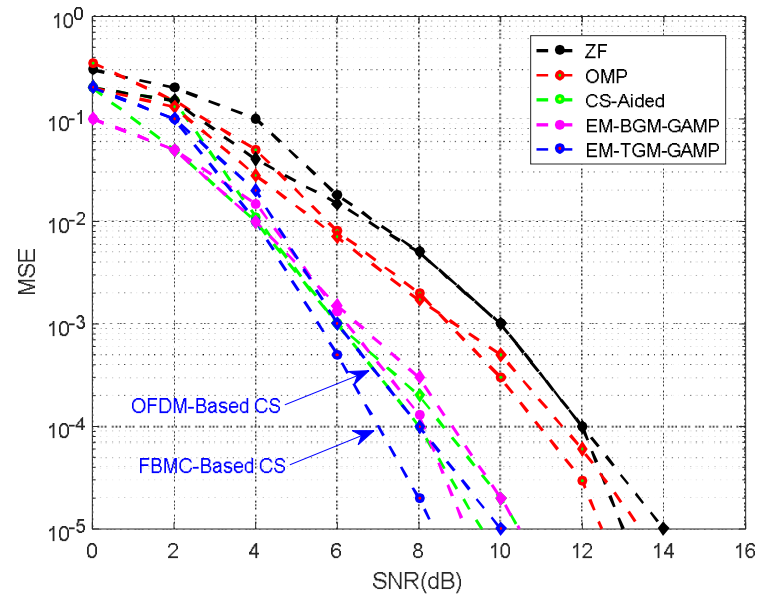

Figure 4: MSE versus SNR performance Comparison of to Compressed sensing based FBMC and OFDM with various schemes.
Moreover, the CS-Aided, OMP and ZF pre-coding scheme show comparatively low error. And the SNR between $8 \mathrm{~dB}$ to $11 \mathrm{~dB}$, the proposed schemes produces better results than ZF and OMP. Throughout the simulation results on figure 4 , the proposed schemes can be obtained the lowest error rate than $\mathrm{ZF}$ and the basic greedy algorithm is called the OMP.

The effectiveness MSE with different algorithms is proved in Figure5. It appears that the suggested schemes are achieved better Signal to noise ratio (SNR) performance compared to the ZF pre-coding. However, the ZF provides the least-norm solution.
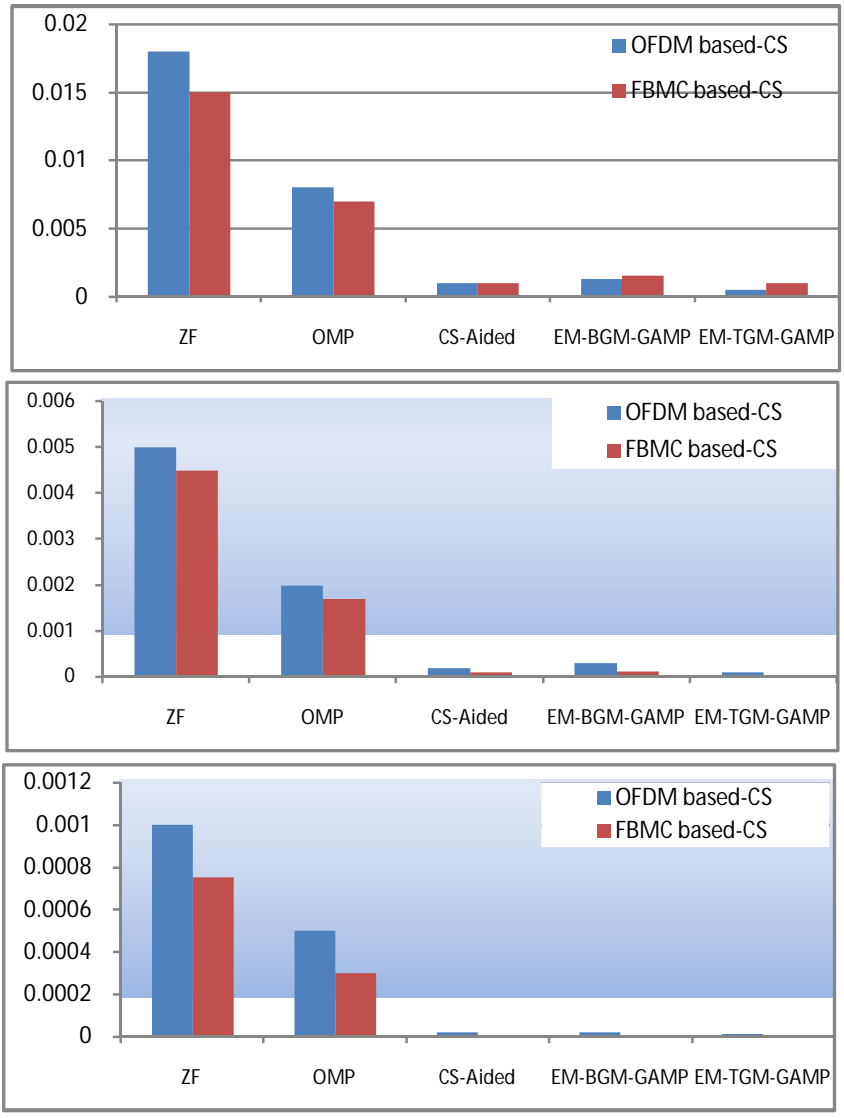

Figure 5: SNR performance of compressed sensing based FBMC and OFDM with various schemes at $6 \mathrm{db}, 8 \mathrm{~dB}$ and $10 \mathrm{~dB}$ respectively.

In order to sustain the same, must have to remove the undesirable characteristics, i.e. perfect normalization is must. In comparison to this, LS based schemes have less error. For SNR between 8-11 dBs, the proposed schemes are better than ZF and as reasonably good performance. Overall, the performed proposed methods have less error than conventional CS techniques.

According to estimate made by comparison, the GMbased CS approaches are easy to determine better training signals. Thus, quality of channel estimate is evaluated by the intended GM-based CS approaches which provides outperform through flagship challenge of the training signals. 


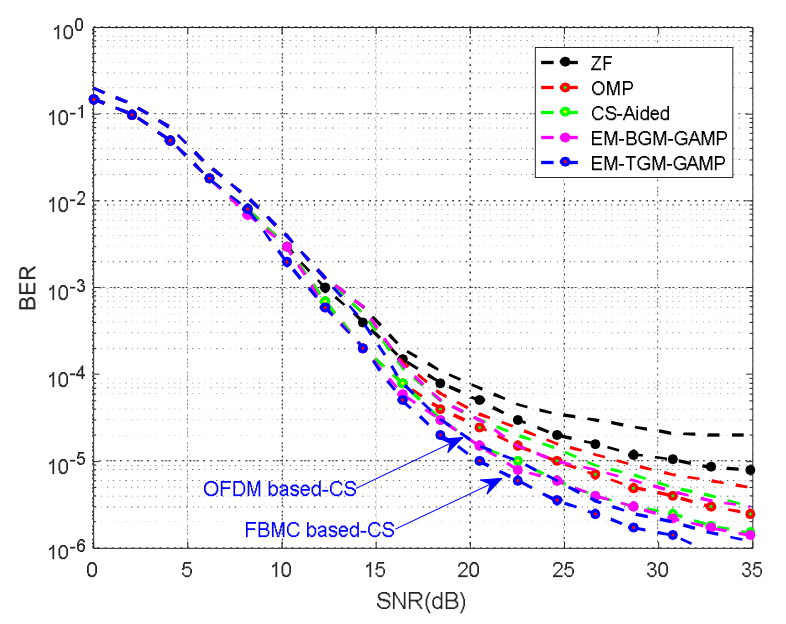

Figure 6: BER versus SNR performance Comparison of to compressed sensing based FBMC and OFDM with various schemes.

Figure 6 indicates the BER versus SNR performance Comparison of to Compressed sensing based FBMC and OFDM with various schemes. The EM-TGM-GAMP algorithm achieved more low BER compared to other CS algorithms in the FBMC environment. The estimation accuracy also can be better by making use of the common sparsity in massive MIMO system.

\section{CONCLUSION}

This work focused on estimate the channel efficacy over OFDM and FBMC based massive MIMO downlink system under various approaches of compressed sensing with Gaussian mixture learning. Particularly the composition of the GAMP technique with the EM iterative methods and they facilitate the less computational complexity through designed pilot approach. Through the continuous support of the CS-Aided approach, the pilot overhead is reduced where insufficient sparsity of the channels. The favorable channel performance is achieved by the use of EM-TGM-GAMP, EM-BGM-GAMP, and CS-Aided schemes. The obtained simulation figures give the truncated GM appears better performance than GB distribution in CS environment. And also provide outperform achievement compared to the $\mathrm{ZF}$ and OMP techniques. The proposed CS framework schemes mitigate the pilot overhead and substantially contribute better performance to estimate channel efficiency.

\section{REFERENCES}

1. Tufvesson, O. Edfors, and T. L. Marzetta, "Massive MIMO for next generation wireless systems," IEEE Commun. Mag., vol. 52, no. 2, pp. 186-195, Feb. 2014.

2. TV. Chen, E. Bjornson "Massive MIMO communications" pp. 77-116, Springer, 2017.

3. Y. Shi, J. Zhang, and K. B. Letaief, "CSI overhead reduction with stochastic beam-forming for cloud radio access networks," in Proc. IEEE Int. Conf. Communications (ICC), Sydney, Australia, Jun. 2014,pp. 5154-5159.

4. S. Nguyen and A. Ghrayeb, "Compressive sensingbased channel estimation for massive multiuser MIMO systems," in Proc. IEEE.

5. X. Gao, F. Tufvesson, and O. Edfors, "Massive MIMO channels -measurements and models," in The 47th Annual Asilomar Conf. Signals, Systems, and Computers, Pacific Grove, California, U.S.A., 2013.

6. D. L. Donoho, A. Maleki, and A. Montanari, "Message passing algorithms for compressed sensing," Proc. Nat. Acad. Sci., vol. 106, no. 45, pp. 18 914-18 919, 2009.

7. S. Rangan, "Generalized approximate message passing for estimation with random linear mixing," in Proc. IEEE Int. Symp. Information Theory (ISIT), Saint Petersburg, Russia, Aug. 2011, pp. 21682172.8.

8. J. P. Vila and P. Schniter, "Expectation-maximization Gaussian-mixture approximate message passing," IEEE Trans. Sig. Process., vol. 61, no. 19, pp. 46584672, Oct. 2013.

9. W. U. Bajwa, J. Haupt, A. M. Sayeed, and R. Nowak, "Compressed channel sensing: A new approach to estimating sparse multipath channels," Proc. IEEE, vol. 98, no. 6, pp. 1058-1076, Jun. 2010.

10. Y. C. Pati, R. Rezaiifar, and P. S. Krishnaprasad, "Orthogonal matching pursuit: Recursive function approximation with applications to wavelet decomposition," in Proc. 27th Annu. Asilomar Conf. Signals, Systems, and Computers, 1993, pp. 40-44.

11. N. Vaswani, "LS-CS-residual (LS-CS): Compressive sensing on least squares residual," IEEE Trans. Signal Process., vol. 58, no. 8, pp. 4108- 4120, Aug. 2010.

12. X. Rao and V. K. N. Lau, "Compressive sensing with prior support quality information and application to massive MIMO channel estimation with temporal correlation," IEEE Trans. Signal Process., vol. 63, no. 18, pp. 4914-4924, Sep. 2015.

13. Farhang, A., Marchetti, N., Doyle, L., and FarhangBoroujeny, B. (2014) Filter bank multicarrier for massive MIMO, in IEEE 80th Vehicular Technology Conference (VTC Fall), pp. 1-7.

14. Y. Barbotin, A. Hormati, S. Rangan, and M. Vetterli, "Estimation of sparse MIMO channels with common support," IEEE Trans. Commun., ol. 60, no. 12, pp. 3705-3716, Dec. 2012.

15. Yin, H., Gesbert, D., Filippou, M., and Liu, Y. (2013) A coordinated approach to channel estimation in large-scale multiple-antenna systems. IEEE J. Select. Areas in Commun.,31(2), 264-273.

16. M. G. Bellanger, "Specification and design of a prototype filter for filter bank based multicarrier transmission," in Proc. 2001 IEEE Int. Conf. acoustics, Speech, and Signal Process., pp. 24172420. 